\title{
One loop renormalizability of the Poisson-Lie sigma models
}

\author{
Galliano VALENT ${ }^{\dagger} \quad$ Ctirad KLIMČÍK
}

Romain SQUELLARI*

†* Laboratoire de Physique Théorique et des Hautes Energies

CNRS, Unité associée URA 280

2 Place Jussieu, F-75251 Paris Cedex 05, France

‡* Institut de Mathématiques de Luminy

CNRS UPR 9016

Case 907, 163 Avenue de Luminy

13288 Marseille Cedex 9, France

\begin{abstract}
We present the proof of the one loop renormalizability in the strict field theoretic sense of the Poisson-Lie $\sigma$-models. The result is valid for any Drinfeld double and it relies solely on the Poisson-Lie structure encoded in the target manifold.
\end{abstract}




\section{Introduction}

At the classical level, the T-duality [10, 11, 4, 1, 7] is an isomorphism between nonlinear $\sigma$-models with geometrically different target manifolds. Or, speaking more precisely, it is the symplectomorphism between the phase spaces of the $\sigma$-models that transforms the Hamiltonian of one model into the Hamiltonian of its dual: it relates right-invariant geometries on a Lie group target $G$ to certain geometries on the dual space $\mathcal{G}^{*}$ of the Lie algebra $\mathcal{G}:=\operatorname{Lie}(G)$. At the quantum level, the status of $T$-duality is much more subtle, mainly because the geometries of the target manifolds generically receive quantum corrections. However, in the particular case of the so called conformal field theories, the $\sigma$-model geometries do not receive such corrections and the $T$-duality can be understood as a unitary transformation relating the spectra and the correlation functions of the quantum $\sigma$-models.

Outside from the conformal points, the original and the dual geometry acquire a dependence on the cut-off. However, if this dependence is such that the geometries remain $T$-dual to each other for every value of the cut-off then $T$-duality is preserved also at the quantum level.

This equivalence problem can be analyzed either in the stringy framework where the $\sigma$-model interacts with matter and gravity or for the pure $\sigma$-model, in which case one has first to prove renormalizability in the strict field theoretic sense and then study the equivalence problem. In the first setting a general proof that the one loop equivalence works was given in [14] provided that the structure constants of $\mathcal{G}$ are traceless. In the second setting, the one-loop renormalizability and the quantum equivalence work without any restriction [3].

The standard non-Abelian $T$-duality has a nontrivial generalization referred to as the Poisson-Lie T-duality [7, 8]. Both the original and the dual geometries of the Poisson-Lie $T$-dualizable $\sigma$-models are derived from the so called Drinfeld double which is a Lie group equipped with some additional structure (cf. Section 2). The one loop equivalence in the stringy framework was shown to work in [2] provided that all the structure constants of the double are traceless. In the restricted field theoretic framework, the one loop renormalizability and compatibility with the renormalization were so far established only for very few special pairs of the mutually dual $\sigma$-models [12, 9]. Those special models live on target manifolds with small dimensions and their geometries can be explicitly calculated in suitable coordinates. At a first sight, it appears to be too difficult a task to deepen this result. Indeed, there are very numerous different Drinfeld doubles (they are far from being all classified) and even for the known Drinfeld doubles (like, for example, simple complex groups), the explicit characterization of the dualizable geometries in terms of coordinates on group manifolds becomes forbiddingly complicated for group targets with dimension 4 and more. In spite of all those obstacles, in this paper we do establish the one loop renormalizability of the Poisson-Lie $\sigma$-models in full generality, i.e. for an arbitrary Drinfeld double. We succeed to obtain this general result mainly because we can express the Ricci tensor in a tractable form just using frames rather than introducing coordinates on the target manifold.

To tell the truth, at the very beginning of our calculation, we did not aim as much as we have eventually obtained. We simply wanted to identify which additional properties a Drinfeld double must possess in order to ensure the one loop renormalizability. To our satisfaction, we find that no such additional properties are needed. In fact, we have been 
impressed how naturally just the basic Poisson-Lie structure (and nothing else) has been sufficient to tame the ultraviolet divergences.

The plan of the paper is as follows. In Section 2, we review the concept of the Drinfeld double and we show how the target geometries are associated to it. In Section 3, the riemannian geometry with torsion is developed and leads to a nice form of the Ricci tensor. This result is then used, in Section 4, to establish the one-loop renormalizability of the Poisson-Lie $\sigma$-model.

\section{Drinfeld doubles and Poisson-Lie T-duality}

Consider a basis $T_{a}, a=1, \ldots, n$ in a vector space $\mathcal{G}$ and the dual basis $\widetilde{T}_{a}$ in the dual space $\widetilde{\mathcal{G}}$. Equip both $\mathcal{G}$ and $\widetilde{\mathcal{G}}$ with Lie algebra structures

$$
\left[T_{a}, T_{b}\right]=f_{a b}^{s} T_{s}, \quad\left[\widetilde{T}^{a}, \widetilde{T}^{b}\right]=\tilde{f}_{s}^{a b} \widetilde{T}^{s}
$$

One says that the Lie algebras $\mathcal{G}$ and $\widetilde{\mathcal{G}}$ are compatible if the following brackets

$$
\left[T_{a}, T_{b}\right]_{\mathcal{D}}=f_{a b}^{s} T_{s}, \quad\left[\widetilde{T}^{a}, \widetilde{T}^{b}\right]_{\mathcal{D}}=\tilde{f}_{s}^{a b} \widetilde{T}^{s} \quad\left[T_{a}, \widetilde{T}^{b}\right]_{\mathcal{D}}=\tilde{f}_{a}^{b s} T_{s}-f_{a s}^{b} \widetilde{T}^{s}
$$

define a Lie algebra structure on the direct sum vector space $\mathcal{D}:=\mathcal{G}+\widetilde{\mathcal{G}}$. In this case, we say that the Lie algebra $\mathcal{D}$ is the Drinfeld double of $\mathcal{G}$ (or, equivalently, of $\widetilde{\mathcal{G}}$ ). Note that the Drinfeld double $\mathcal{D}$ comes equipped with an $A d$-invariant bilinear form $\langle., .\rangle_{\mathcal{D}}$ defined as

$$
\left\langle T_{a}, \widetilde{T}^{b}\right\rangle_{\mathcal{D}}=\delta_{a}^{b}, \quad\left\langle T_{a}, T_{b}\right\rangle_{\mathcal{D}}=\left\langle\widetilde{T}^{a}, \widetilde{T}^{b}\right\rangle_{\mathcal{D}}=0 .
$$

Consider the connected and simply connected group $D$ the Lie algebra of which is $\mathcal{D}$ and the subgroups $G$ and $\widetilde{G}$ of $D$ corresponding to the subalgebras $\mathcal{G} \subset \mathcal{D}$ and $\widetilde{\mathcal{G}} \subset \mathcal{D}$, respectively. The group $D$ is called the Drinfeld double of $G$ (or of $\widetilde{G}$ ).

In what follows, we shall be often using the matrices of the adjoint action of $D$ on $\mathcal{D}$ in the basis $T_{a}, \widetilde{T}^{b}$ :

$$
\begin{gathered}
A d_{g} \widetilde{T}^{a} \equiv l^{-1} T l=B^{a s}(g) T s+A_{s}^{a}\left(g^{-1}\right) \widetilde{T}^{s}, \quad g \in G \\
A d_{\tilde{g}} T_{a}=\widetilde{B}_{a s}(\tilde{g}) \widetilde{T}^{s}+\widetilde{A}_{a}^{s}\left(\tilde{g}^{-1}\right) T_{s}, \quad \tilde{g} \in \widetilde{G}
\end{gathered}
$$

We can write those matrices also in terms of the bilinear form (1i) as

$$
\begin{array}{ll}
A_{a}^{b}(g):=\left\langle A d_{g} T_{a}, \widetilde{T}^{b}\right\rangle, & B^{a b}(g):=\left\langle A d_{g} \widetilde{T}^{a}, \widetilde{T}^{b}\right\rangle ; \\
\widetilde{A}_{a}^{b}(\tilde{g}):=\left\langle T_{a}, A d_{\tilde{g}} \widetilde{T}^{b}\right\rangle, & \widetilde{B}_{a b}(\tilde{g}):=\left\langle A d_{\tilde{g}} T_{a}, T_{b}\right\rangle .
\end{array}
$$

It turns out that the algebraic structures that we have introduced so far can be used to define certain Poisson brackets of functions on the groups $G$ and $\widetilde{G}$ :

$$
\left\{f_{1}, f_{2}\right\}(g):=\Pi^{a b}(g) \nabla_{a} f_{1}(g) \nabla_{b} f_{2}(g), \quad\left\{f_{1}, f_{2}\right\}^{*}(\tilde{g}):=\widetilde{\Pi}_{a b}(\tilde{g}) \nabla^{a} \tilde{f}_{1}(\tilde{g}) \nabla^{b} f_{2}^{*}(\tilde{g}),
$$

where the right-invariant vector fields $\nabla_{a}$ and $\widetilde{\nabla}^{a}$ on $G$ and $\widetilde{G}$ are defined by

$$
\nabla_{a} f(g):=\left.\frac{d}{d s} f\left(e^{s T_{a}} g\right)\right|_{s=0}, \quad \widetilde{\nabla}^{a} \tilde{f}(\tilde{g}):=\left.\frac{d}{d s} \tilde{f}\left(e^{s \widetilde{T}^{a}} \tilde{g}\right)\right|_{s=0}
$$


and the antisymmetric matrix-valued functions $\Pi^{a b}(g)$ and $\widetilde{\Pi}_{a b}(\tilde{g})$ are given by

$$
\Pi^{a b}(g):=-B(g)_{a s} A_{s}^{b}\left(g^{-1}\right), \quad g \in G ; \quad \widetilde{\Pi}_{a b}(\tilde{g}):=-\widetilde{B}_{a s}(\tilde{g}) \widetilde{A}_{b}^{s}\left(\tilde{g}^{-1}\right), \quad \tilde{g} \in \widetilde{G} .
$$

The Poisson structures on $G$ and $\widetilde{G}$ turn out to satisfy the so-called cocycle conditions in the $\mathcal{G} \wedge \mathcal{G}$ and $\widetilde{\mathcal{G}} \wedge \widetilde{\mathcal{G}}$-valued group cohomologies of $G$ and $\widetilde{G}$, respectively:

$$
\Pi(h g)=\Pi(h)+A d_{h} \Pi(g), \quad g, h \in G ; \quad \widetilde{\Pi}(\tilde{h} \tilde{g})=\widetilde{\Pi}(\tilde{h})+A d_{\tilde{h}} \widetilde{\Pi}(\tilde{g}), \quad \tilde{g}, \tilde{h} \in \widetilde{G} .
$$

Here we have set

$$
\Pi(g):=\Pi^{a b}(g) T_{a} \otimes T_{b}, \quad \widetilde{\Pi}(\tilde{g}):=\widetilde{\Pi}_{a b}(\tilde{g}) \widetilde{T}^{a} \otimes \widetilde{T}^{b} .
$$

Consider an invertible matrix $M^{a b}$ and the maps $E_{M}: G \rightarrow \mathcal{G} \otimes \mathcal{G}$ and $\widetilde{E}_{M}: \widetilde{\mathcal{G}} \rightarrow \widetilde{\mathcal{G}} \otimes \widetilde{\mathcal{G}}$ defined by

$$
\begin{aligned}
E_{M}(g) & :=E_{M}(g)^{a b} T_{a} \otimes T_{b} ; & \widetilde{E}_{M}(\tilde{g}) & :=\widetilde{E}_{M}(\tilde{g})_{a b} \widetilde{T}^{a} \otimes \widetilde{T}^{b}, \\
E_{M}(g)^{a b} & :=M^{a b}+\Pi^{a b}(g) ; & \widetilde{E}_{M}(\tilde{g})_{a b} & :=\left(M^{-1}\right)_{a b}+\widetilde{\Pi}_{a b}(\tilde{g}) .
\end{aligned}
$$

The Poisson-Lie $T$-duality then establishes the isomorphism between $\sigma$-models living on the targets $G$ and $\widetilde{G}$. These models are completely specified by the maps $E_{M}$ and $\widetilde{E}_{M}$. Their respective field configurations are smooth maps $g: W \rightarrow G$ and $\tilde{g}: W \rightarrow \widetilde{G}$, where $W$ is the two-dimensional world-sheet, and their respective dynamics are determined by the least action principles:

$$
\begin{aligned}
& S(g)=\int_{W}\left(E_{M}(g)^{-1}, R(g)_{+} \otimes R(g)_{-}\right) d \xi^{+} d \xi^{-} \\
& \widetilde{S}(\tilde{g})=\int_{W}\left(\widetilde{E}_{M}(\tilde{g})^{-1}, R(\tilde{g})_{+} \otimes R(\tilde{g})_{-}\right) d \xi^{+} d \xi^{-} .
\end{aligned}
$$

Here $(.,$.$) is the duality pairing between \mathcal{G} \otimes \mathcal{G}$ and $\widetilde{\mathcal{G}} \otimes \widetilde{\mathcal{G}}, \xi^{+}, \xi^{-}$are the light-cone coordinates on $W, R(g)_{+} d \xi^{+}+R(g)_{-} d \xi^{-}$denotes the pull-back of the right-invariant Maurer-Cartan form on $G$ by the map $g: W \rightarrow G$ and $E_{M}^{-1}: G \rightarrow \widetilde{\mathcal{G}} \otimes \widetilde{\mathcal{G}}$ is inverse to $E_{M}$, i.e.

$$
E_{M}(g)\left(., E_{M}(g)^{-1}(., u)\right)=u, \quad \forall g \in G, \quad \forall u \in \mathcal{G} .
$$

Similarly, $R(\tilde{g})_{+} d \xi^{+}+R(\tilde{g})_{-} d \xi^{-}$denotes the pull-back of the right-invariant Maurer-Cartan form on $\widetilde{G}$ by the map $\tilde{g}: W \rightarrow \widetilde{G}$ and $\widetilde{E}_{M}^{-1}: \widetilde{G} \rightarrow \mathcal{G} \otimes \mathcal{G}$ is inverse to $\widetilde{E}_{M}$.

If we introduce some local coordinates $X^{\mu}$ e.g. on the target $G$, any field configuration $g$ can be locally viewed as a collection of real functions $X^{\mu}\left(\xi^{+}, \xi^{-}\right)$in terms of which the action (6) can be locally rewritten as

$$
S=\int_{W}\left(g_{\mu \nu}(X)+h_{\mu \nu}(X)\right) \partial_{\xi^{+}} X^{\mu} \partial_{\xi^{-}} X^{\nu} d \xi^{+} d \xi^{-}
$$

Here $g_{\mu \nu}$ is a symmetric tensor interpreted as a metric on $G$ and $h_{\mu \nu}$ is an antisymmetric tensor interpreted as a torsion potential on $G$. Thus we see that any choice of the map $E_{M}: G \rightarrow \mathcal{G} \otimes \mathcal{G}$ defines an $M$-dependent geometry on $G$.

We note one crucial fact: the moduli space of the dual pairs of the $\sigma$-models (6), (7) associated to a given Drinfeld double $D$ is finite-dimensional since it is parametrized 
by the invertible matrices $M$. If we wish that the quantum corrections do not spoil the $T$-dualizability, all ultraviolet divergences must be eliminated just by a suitable cut-off dependence of the matrix $M$. In other words, if we interpret the entries of the matrix $M$ as the coupling constants, we simply require the renormalizability of the model (6) in the standard field theoretic sense of this term. As we shall see in the next section, this is precisely what happens.

The matrices $\Pi^{i j}(g)$ and $\widetilde{\Pi}_{i j}(\tilde{g})$ appear explicitly in the $\sigma$-model Lagrangians on the targets $G$ and $\widetilde{G}$, it is therefore obvious that the countertems needed to cancel the ultraviolet divergences must depend algebro-differentially on them. Actually, if $\Pi$ and $\widetilde{\Pi}$ were generic matrix-valued functions on $G$ and $\widetilde{G}$, the dependence of the counterterms on them would be too complicated to ensure renormalizability, However, due to their special definitions, $\Pi$ and $\widetilde{\Pi}$ satisfy two crucial identities which, quite remarkably, are sufficient to disentangle the counterterm structure and ensure renormalizability. They were first derived in [13] in a completely algebraic way, but it is perhaps more insightful to understand these relations as resulting from the Poisson-Lie geometry, as shown in [6]. The first of those identities is the direct consequence of the cocycle condition (3) for $h$ and $\tilde{h}$ respectively close to the group units of $G$ and $\widetilde{G}$. It reads

$$
\begin{aligned}
\nabla_{c} \Pi^{a b}(g) & =\tilde{f}_{c}^{a b}-f_{c s}^{a} \Pi^{b s}(g)+f_{c s}^{b} \Pi^{a s}(g) \\
\widetilde{\nabla}^{c} \widetilde{\Pi}_{a b}(\tilde{g}) & =f_{a b}^{c}-\tilde{f}_{a}^{c s} \widetilde{\Pi}_{b s}(\tilde{g})+\tilde{f}_{a}^{c s} \widetilde{\Pi}^{a s}(\tilde{g})
\end{aligned}
$$

The second identity is nothing but the Jacobi identity for the Poisson brackets on $G$ and on $\widetilde{G}$ :

$$
\begin{aligned}
& \Pi^{s a}(g) \nabla_{s} \Pi^{b c}(g)+f_{s t}^{a} \Pi^{b s}(g) \Pi^{c t}(g)+c p(a, b, c)=0 \\
& \widetilde{\Pi}^{s a}(\tilde{g}) \widetilde{\nabla}^{s} \widetilde{\Pi}_{b c}(\tilde{g})+\tilde{f}_{a}^{s t} \widetilde{\Pi}^{b s}(\tilde{g}) \widetilde{\Pi}^{c t}(\tilde{g})+c p(a, b, c)=0
\end{aligned}
$$

where $c p(a, b, c)$ means circular permutation of the indices involved. As a consequence of (91), (10), (11) and (12), we obtain

$$
\begin{aligned}
& f_{s t}^{a} \Pi^{b s}(g) \Pi^{c t}(g)+\tilde{f}_{s}^{a b} \Pi^{c s}(g)+c p(a, b, c)=0 . \\
& \tilde{f}_{a}^{s t} \widetilde{\Pi}_{b s}(\tilde{g}) \widetilde{\Pi}_{c t}(\tilde{g})+f_{a b}^{s} \widetilde{\Pi}^{c s}(\tilde{g})+c p(a, b, c)=0 .
\end{aligned}
$$

\section{The geometry of Poisson-Lie $\sigma$-models}

\subsection{Geometry with torsion}

The form (6) of the model gives a prominent role to the right-invariant frames:

$$
d g g^{-1}=R^{a}(g) T_{a}, \quad d R^{a}(g)=\frac{1}{2} f_{b c}^{a} R^{b}(g) \wedge R^{c}(g)
$$

leading us to introduce

$$
G_{a b} R^{a}(g) R^{b}(g), \quad G_{a b}=g_{(a b)}+h_{[a b]},
$$

where $g$ will be a riemannian metric and $h$ the torsion potential. ¿From $h$ we get the torsion 3-form according to

$$
H=\frac{1}{2} h_{a b} R^{a} \wedge R^{b} \Rightarrow T=d H=\frac{1}{3 !} T_{a b c} R^{a} \wedge R^{b} \wedge R^{c}
$$


and we will need also

$$
T^{a}=\frac{1}{2} T_{b c}^{a} R^{b} \wedge R^{c}, \quad T_{b c}^{a}=g^{a s} T_{s b c} .
$$

Putting coordinates $\left\{X^{\mu}\right\}$ on the group we recover the metric and the torsion potential defined in (8) by

$$
g_{\mu \nu}=g_{a b} R_{\mu}^{a} R_{\nu}^{b}, \quad h_{\mu \nu}=h_{a b} R_{\mu}^{a} R_{\nu}^{b},
$$

and the symmetric connection $\nabla$ defined as usual by

$$
\nabla_{\mu} v_{\nu}=\partial_{\mu} v_{\nu}-\gamma_{\mu \nu}^{\sigma} v_{\sigma}, \quad \gamma_{\mu \nu}^{\alpha}=\frac{1}{2} g^{\alpha \sigma}\left(\partial_{\mu} g_{\nu \sigma}+\partial_{\nu} g_{\mu \sigma}-\partial_{\sigma} g_{\mu \nu}\right)
$$

One can define two connections with torsion:

$$
D_{\mu}^{ \pm} v_{\nu}=\nabla_{\mu} v_{\nu} \pm \frac{1}{2} T_{\mu \nu}^{\sigma} v_{\sigma}, \quad D_{\alpha}^{ \pm} g_{\mu \nu}=0,
$$

both compatible with the metric.

The spin connections $\Omega^{ \pm}$and their structure equations are

$$
D_{\mu}^{ \pm} R_{\nu}^{a}=-\Omega_{b \mu}^{ \pm a} R_{\nu}^{b}, \quad d R^{a}+\Omega^{ \pm a}{ }_{s} \wedge R^{s} \pm T^{a}=0 .
$$

The explicit formula for $\Omega^{-}$is 1

$$
\Omega_{a b c}^{-}=\frac{1}{2}\left(\widetilde{\partial}_{b} G_{a c}+\widetilde{\partial}_{c} G_{b a}-\widetilde{\partial}_{a} G_{b c}\right)+\frac{1}{2}\left(-f_{a b}^{s} G_{s c}+f_{c a}^{s} G_{b s}-f_{c b}^{s} G_{a s}\right),
$$

Raising one index, we have

$$
\Omega_{b c}^{ \pm a}=g^{a s} \Omega_{s b c}^{ \pm}, \quad \Omega_{b c}^{+a}-\Omega_{c b}^{-a}=f_{b c}^{a},
$$

as well as

$$
\Omega_{a, s}^{-s}=\Omega_{a, s}^{+s}=\widetilde{\partial}_{a} \ln (\sqrt{\operatorname{det} g})+f_{a s}^{s} .
$$

Working with the connection $\Omega^{-}$, we will define the curvature as

$$
R_{b}^{a}=d \Omega_{b}^{-a}+\Omega_{s}^{-a} \wedge \Omega_{b}^{-s}=\frac{1}{2} R_{b, s t}^{a} R^{s} \wedge R^{t},
$$

which gives

$$
R_{b, c d}^{a}=\widetilde{\partial}_{c} \Omega_{b d}^{-a}-\widetilde{\partial}_{d} \Omega_{b c}^{-a}+\Omega_{b s}^{-a} f_{c d}^{s}+\Omega_{s c}^{-a} \Omega_{b d}^{-s}-\Omega_{u d}^{-a} \Omega_{b c}^{-u} .
$$

Defining the Ricci tensor and scalar as 2

$$
R i c_{a b}=R_{a, s b}^{s}, \quad R=g^{a b} \operatorname{Ric}_{a b},
$$

and using relation (24) we end up with

$$
R i c_{a b}=\widetilde{\partial}_{s} \Omega_{a b}^{-s}-\Omega_{a, t}^{-s} \Omega_{b, s}^{+t}-\mathcal{D}_{b} v_{a}, \quad v_{a}=\Omega_{a, s}^{-s},
$$

using the frame covariant derivative

$$
\mathcal{D}_{b} v_{a}=\widetilde{\partial}_{b} v_{a}-\Omega_{a b}^{-s} v_{s} .
$$

\footnotetext{
${ }^{1}$ To avoid confusion we change $\nabla_{a}$, defined by (2), into $\widetilde{\partial}_{a}$ defined by $\partial_{\mu}=R_{\mu}^{a} \widetilde{\partial}_{a}$.

${ }^{2}$ The sphere has positive curvature.
} 


\subsection{Metric and torsion potential}

As seen in Section 2 the classical action is

$$
S=\int(M+\Pi(g))_{a b}^{-1} R_{+}^{a}(g) R_{-}^{b}(g) d \xi^{+} d \xi^{-} .
$$

Comparing this expression with (16) we can write

$$
G_{a b}=(M+\Pi)_{a b}^{-1}=g_{a b}+h_{a b}, \quad \Gamma^{a b}=M^{a b}+\Pi^{a b}, \quad G_{a s} \Gamma^{s b}=\Gamma^{b s} G_{s a}=\delta_{a}^{b} .
$$

The riemannian metric can be written in two ways

$$
g=\frac{1}{2}\left(G+G^{t}\right)=G M_{S} G^{t}=G^{t} M_{S} G, \quad M_{S}=\frac{1}{2}\left(M+M^{t}\right),
$$

as well as its inverse

$$
g^{-1}=\Gamma^{t} M_{S}^{-1} \Gamma=\Gamma M_{S}^{-1} \Gamma^{t}
$$

\subsection{The spin connection}

Let us write relation (23) as

$$
2 \Omega_{a b c}^{-}=\left(\widetilde{\partial}_{b} G_{a c}+f_{b c}^{s} G_{a s}\right)+\left(\widetilde{\partial}_{c} G_{b a}+f_{c a}^{s} G_{b s}\right)-\left(\widetilde{\partial}_{a} G_{b c}+f_{a b}^{t} G_{t c}\right) .
$$

Using the identity (9) we can combine, in the first term of (35), the two pieces to obtain

$$
\widetilde{\partial}_{b} G_{a c}+f_{b c}^{s} G_{a s}=-G_{a s} X_{b}^{s t} G_{t c}
$$

with

$$
X_{c}^{a b}=\widetilde{F}_{c}^{a b}-\Pi^{a s} f_{s c}^{b}, \quad \widetilde{F}_{c}^{a b}=\tilde{f}_{c}^{a b}-f_{b s}^{a} M^{s c} .
$$

Similar computations for the remaining terms give

$$
2 \Omega_{a b c}^{-}=-G_{a s} X_{b}^{s t} G_{t c}-G_{b s} X_{c}^{s t} G_{t a}+G_{b s} Y_{a}^{s t} G_{t c}
$$

with

$$
Y_{c}^{a b}=\widehat{F}_{c}^{a b}+f_{c s}^{a} \Pi^{s b}, \quad \widehat{F}_{c}^{a b}=\tilde{f}_{c}^{a b}+M^{a s} f_{s c}^{b} .
$$

Raising the first index of the connection, we have

$$
2 \Omega_{b c}^{-a}=-g^{a \alpha} G_{\alpha s} X_{b}^{s t} G_{t c}-G_{b s} X_{c}^{s t} G_{t \alpha} g^{\alpha a}+g^{a \alpha} Y_{\alpha}^{s t} G_{b s} G_{t c} .
$$

Using $g^{a b}=\left(\Gamma M_{S}^{-1} \Gamma^{t}\right)^{a b}$ one gets

$$
g^{a \alpha} G_{\alpha s}=2 \delta_{s}^{a}-\left(\Gamma M_{S}^{-1}\right)^{a}{ }_{s}, \quad G_{t \alpha} g^{\alpha a}=\left(\Gamma M_{S}^{-1}\right)^{a}{ }_{t},
$$

leading to

$$
2 \Omega_{b c}^{-a}=-2 X_{b}^{a s} G_{s c}+\left(\Gamma M_{S}^{-1}\right)^{a}{ }_{v}\left(X_{u}^{v t} \Gamma^{s u}-X_{u}^{s v} \Gamma^{u t}+Y_{u}^{s t} \Gamma^{u v}\right) G_{b s} G_{t c} .
$$

The second term in the right hand side is a polynomial of degree 2 in the $\Pi$ 's. Getting rid of the quadratic terms upon use of the identity (13) all the terms linear in $\Pi$ cancel out and

$$
\Omega_{b c}^{-a}=-X_{b}^{a s} G_{s c}+G_{b s} \Gamma^{a u} \widehat{\mathcal{F}}_{u}^{s t} G_{t c}
$$


with

$$
\widehat{\mathcal{F}}_{u}^{s t}=\frac{1}{2}\left(M_{S}^{-1}\right)_{u v}\left(\widehat{F}_{a}^{s t} M^{a v}+\widetilde{F}_{a}^{v t} M^{s a}-\widetilde{F}_{a}^{s v} M^{a t}\right) .
$$

For further use, let us define

$$
\widetilde{\mathcal{F}}_{u}^{s t}=\frac{1}{2}\left(M_{S}^{-1}\right)_{u v}\left(\widetilde{F}_{a}^{s t} M^{v a}+\widehat{F}_{a}^{s v} M^{a t}-\widehat{F}_{a}^{v t} M^{s a}\right)
$$

and mention the identities

$$
\widehat{F}_{c}^{a b}+\widetilde{F}_{c}^{a b}-\widehat{\mathcal{F}}_{c}^{a b}-\widetilde{\mathcal{F}}_{c}^{a b}=\tilde{f}_{c}^{a b}, \quad \widetilde{\partial}_{s} \Pi^{a b}=X_{s}^{a b}+f_{s u}^{a} \Gamma^{u b}=Y_{s}^{a b}-\Gamma^{a u} f_{u s}^{b} .
$$

Using these relations and (24) and (43) we can write

$$
\Omega_{b c}^{+a}=-\widetilde{\partial}_{c} \Pi^{a s} G_{s b}+G_{c s} \Gamma^{a u} \widehat{\mathcal{F}}_{u}^{s t} G_{t b} .
$$

If, instead of relations (41), we use for the inverse metric its form $g^{a b}=\left(\Gamma^{t} M_{S}^{-1} \Gamma\right)^{a b}$, then instead of (41) we have

$$
g^{a \alpha} G_{\alpha s}=\left(\Gamma^{t} M_{S}^{-1}\right)^{a}{ }_{s}, \quad G_{t \alpha} g^{\alpha a}=2 \delta_{t}^{a}-\left(\Gamma^{t} M_{S}^{-1}\right)^{a}{ }_{t},
$$

leading this time to

$$
2 \Omega_{b, c}^{-a}=-2 X_{c}^{s a} G_{b s}+\left(\Gamma^{t} M_{S}^{-1}\right)_{v}^{a}\left(Y_{u}^{s t} \Gamma^{v u}-X_{u}^{v t} \Gamma^{s u}+X_{u}^{s v} \Gamma^{u t}\right) G_{b s} G_{t c} .
$$

Computations similar to the ones leading to (43) give then

$$
\Omega_{b, c}^{-a}=-X_{c}^{s a} G_{b s}+G_{b s}\left(\Gamma^{t}\right)^{a u}\left(Y_{u}^{s t}-\widehat{\mathcal{F}}_{u}^{s t}\right) G_{t c} .
$$

\subsection{The Ricci tensor}

Let us start from (29):

$$
R i c_{a b}=\widetilde{\partial}_{s} \Omega_{a b}^{-s}-\Omega_{a, t}^{-s} \Omega_{b, s}^{+t}-\mathcal{D}_{b} v_{a}, \quad v_{a}=\widetilde{\partial}_{a}(\ln \sqrt{\operatorname{det} g})+f_{a s}^{s} .
$$

In the first term we use (43), while in the product we use (50) for $\Omega^{-}$and (47) for $\Omega^{+}$. The resulting expression is either cubic or quadratic in $G$. We will write it as

$$
\operatorname{Ric}_{a b}=G_{a \alpha} \mathcal{L}^{\alpha \beta} G_{\beta b}+G_{a \alpha} \mathcal{M}^{\alpha \beta, \lambda \mu} G_{\lambda \mu} G_{\beta b}
$$

The quantities $\mathcal{L}($ resp. $\mathcal{M}$ ) are quadratic (resp. cubic) with respect to $\Pi$. Let us first explain how one can get rid of the terms cubic in $G$. Let us consider:

$$
\mathcal{M}^{\alpha \beta, \lambda \mu} G_{\lambda \mu}=\left(\Gamma^{\alpha s} X_{s}^{t \lambda}+Y_{s}^{\alpha \lambda} \Gamma^{s t}-2 \widehat{\mathcal{F}}_{s}^{\alpha \lambda} M_{S}^{s t}\right) G_{\lambda \mu} \widetilde{\partial}_{t} \Pi^{\mu \beta} \equiv H^{\alpha t \lambda} G_{\lambda \mu} \widetilde{\partial}_{t} \Pi^{\mu \beta}
$$

The terms in $H$ are quadratic in $\Pi$ and can be reduced as follows. Using the identity (13) the terms quadratic in $\Pi$ reduce to

$$
f_{u v}^{t} \Pi^{\alpha v} \Pi^{u \lambda}=f_{u v}^{t} \Pi^{\alpha v} \Gamma^{u \lambda}-f_{u v}^{t} \Pi^{\alpha v} M^{u \lambda}
$$


and the first piece, when multiplied by $G_{\lambda \mu}$, gives a contribution quadratic in $\mathrm{G}$. Then the terms remaining in $H$ are just linear in $\Pi$ and they combine to

$$
\widetilde{F}_{s}^{\alpha t} \Pi^{s \lambda}=\widetilde{F}_{s}^{\alpha t} \Gamma^{s \lambda}-\widetilde{F}_{s}^{\alpha t} M^{s \lambda}
$$

The first piece gives another contribution quadratic in $\mathrm{G}$. The remaining terms in $H$ are now independent of $\Pi$ and vanish as a consequence of (44).

Gathering all of the pieces quadratic in $G$ we get, apart from a few trivial cancellations

$$
\widetilde{\partial}_{s} \Omega_{a b}^{-s}-\Omega_{a, t}^{-s} \Omega_{b, s}^{+t}=G_{a \alpha}\left\{\left(f_{t u}^{\beta} \Gamma^{\alpha u}+\widehat{\mathcal{F}}_{t}^{\alpha \beta}\right) \widetilde{\partial}_{s} \Pi^{s t}-\left(Y_{t}^{\alpha s}-\widehat{\mathcal{F}}_{s}^{t \beta}+f_{u t}^{\alpha} \Gamma^{u s}\right) \widehat{\mathcal{F}}_{s}^{t \beta}\right\} G_{\beta b} .
$$

The second term, in the right hand part simplifies, using (46), to

$$
\widetilde{\partial}_{s} \Omega_{a b}^{-s}-\Omega_{a, t}^{-s} \Omega_{b, s}^{+t}=G_{a \alpha}\left\{\left(f_{t u}^{\beta} \Gamma^{\alpha u}+\widehat{\mathcal{F}}_{t}^{\alpha \beta}\right) \widetilde{\partial}_{s} \Pi^{s t}+\widetilde{\mathcal{F}}_{t}^{\alpha s} \widehat{\mathcal{F}}_{s}^{t \beta}\right\} G_{\beta b} .
$$

To get rid of the residual $\Pi$ dependence, we will consider the vector field $w_{a}=G_{a s} \widetilde{\partial}_{t} \Pi^{t s}$. Using (30) and (50) we get

$$
\mathcal{D}_{b} w_{a}=G_{a \alpha}\left\{\left(\widetilde{\partial}_{s t}^{2} \Pi^{t \alpha}-f_{s u}^{\alpha} \widetilde{\partial}_{t} \Pi^{t u}\right) \Gamma^{s \beta}-\left(Y_{s}^{\alpha \beta}-\widehat{\mathcal{F}}_{s}^{\alpha \beta}\right) \widetilde{\partial}_{t} \Pi^{t s}\right\} G_{\beta b} .
$$

The relation (80), proved in appendix A, shows that the coefficient of $\Gamma^{s \beta}$ in the previous relation does vanish. So we can use (57) in the following way

$$
G_{a \alpha} \widehat{\mathcal{F}}_{s}^{\alpha \beta} \widetilde{\partial}_{t} \Pi^{t s} G_{\beta b}=\mathcal{D}_{b} w_{a}+G_{a \alpha} \widetilde{\partial}_{t} \Pi^{t s} Y_{s}^{\alpha \beta} G_{\beta b}
$$

to transform (156) into

$$
\widetilde{\partial}_{s} \Omega_{a b}^{-s}-\Omega_{a, t}^{-s} \Omega_{b, s}^{+t}=\mathcal{D}_{b} w_{a}+G_{a \alpha}\left\{\widetilde{\mathcal{F}}_{t}^{\alpha s} \widehat{\mathcal{F}}_{s}^{t \beta}+\widetilde{\partial}_{s} \Pi^{s t} \widetilde{\partial}_{t} \Pi^{\alpha \beta}\right\} G_{\beta b} .
$$

The residual term quadratic in the derivatives of $\Pi$ does vanish as a consequence of the relation (79) proved in appendix A. Therefore we have

$$
\left\{\begin{array}{l}
\operatorname{Ric}_{a b}=G_{a \alpha} r_{0}^{\alpha \beta} G_{\beta b}+\mathcal{D}_{b}\left(w_{a}-v_{a}\right), \\
r_{0}^{\alpha \beta}=\widetilde{\mathcal{F}}_{t}^{\alpha s} \widehat{\mathcal{F}}_{s}^{t \beta}, \\
v_{a}=\widetilde{\partial}_{a}(\ln \sqrt{\operatorname{det} g})+f_{a s}^{s}, \quad w_{a}=G_{a s} \widetilde{\partial}_{t} \Pi^{t s} .
\end{array}\right.
$$

Of course the Ricci tensor is uniquely defined, however the writing of the result is not unique for the following reason. Let us consider a vector of the form $W_{a}=G_{a \alpha} \xi^{\alpha}$. Using the connection given by (50), we get

$$
\mathcal{D}_{b} W_{a}=\left(\widetilde{\partial}_{s} \xi^{\alpha} \Gamma^{s \beta}-\xi^{s}\left(f_{u s}^{\alpha} \Gamma^{u \beta}+Y_{s}^{\alpha \beta}-\widehat{\mathcal{F}}_{s}^{\alpha \beta}\right)\right) G_{a \alpha} G_{\beta b}
$$

The second term simplifies and if we consider coordinate independent $\xi^{\alpha}$ we get

$$
\mathcal{D}_{b} W_{a}=-\xi^{s} \widetilde{\mathcal{F}}_{s}^{\alpha \beta} G_{a \alpha} G_{\beta b} .
$$

This generates an ambiguity since we can write

$$
R i c_{a b}=G_{a \alpha} r^{\alpha \beta} G_{\beta b}+\mathcal{D}_{b}\left(w_{a}+W_{a}-v_{a}\right), \quad r^{\alpha \beta}=r_{0}^{\alpha \beta}+\xi^{t} \widetilde{\mathcal{F}}_{t}^{\alpha \beta},
$$

for any coordinate independent $\xi^{\alpha}$. ¿From the previous result, by the obvious duality substitutions, one can easily get the Ricci tensor for the dual model. 


\section{One loop renormalizability}

We started from the classical action

$$
S=\frac{1}{2} \int G_{a b} R_{+}^{a} R_{-}^{b} d \xi^{+} d \xi^{-}
$$

The one loop counterterm was first computed by Fridling and van de Ven [5] for $\sigma$-models with general torsion. Their result is

$$
\frac{1}{4 \pi \epsilon} \int \operatorname{Ric}_{a b} R_{+}^{a} R_{-}^{b} d \xi^{+} d \xi^{-}, \quad \epsilon=2-d
$$

where the Ricci tensor is computed with the $D^{-}$connection.

Renormalizability in the strict field theoretic sense requires that these divergences have to be absorbed by field independent deformations of the coupling constants $M^{s t}$ and possibly a non-linear field renormalization of the fields $X^{\mu}$. We have first to define the coupling constants of the theory. Since we are analyzing the most general Poisson-Lie model, which is built up from an arbitrary constant matrix $M$, we will take as independent coupling constants all the matrix elements of $M$. When working in coordinates, we have to check the relations

$$
\operatorname{Ric}_{(\mu \nu)}=\chi^{s t} \frac{\partial}{\partial M^{s t}} g_{\mu \nu}+\nabla_{(\mu} u_{\nu)}, \quad \operatorname{Ric}_{[\mu \nu]}=\chi^{s t} \frac{\partial}{\partial M^{s t}} h_{\mu \nu}+T_{\mu \nu}^{\sigma} u_{\sigma}+\partial_{[\mu} U_{\nu]},
$$

for some vectors $u, U$ and coordinate independent $\chi^{s t}$. Adding these relations we can write

$$
R i c_{\mu \nu}=\chi^{s t} \frac{\partial}{\partial M^{s t}} G_{\mu \nu}+D_{\nu}^{-} u_{\mu}+\partial_{[\mu}(u+U)_{\nu]}
$$

and since the frames are independent of the parameters of the matrix $M$, this relation becomes, using frame components

$$
R i c_{a b}=\chi^{s t} \frac{\partial}{\partial M^{s t}} G_{a b}+\mathcal{D}_{b} u_{a}+\widetilde{\partial}_{[a}(u+U)_{b]}+f_{a b}^{s}(u+U)_{s} .
$$

Since relation (63) writes

$$
R i c_{a b}=G_{a \alpha} r^{\alpha \beta} G_{\beta b}+\mathcal{D}_{b}\left(w_{a}+W_{a}-v_{a}\right),
$$

we obtain

$$
\chi^{a b}=-r^{a b}, \quad U_{a}=-u_{a}, \quad u_{a}=w_{a}+W_{a}-v_{a},
$$

and this concludes the one loop renormalizability proof.

Let us observe that the non-uniqueness of $r^{a b}$ explained in the previous section has

for effect that the renormalization constants themselves are not uniquely defined. This phenomenon had already been observed in [9].

\section{A Some identities}

We have gathered in this Appendix the proofs of various identities used in the article. 


\section{A.1 Derivative of the bivector}

Let us start from the relation which defines the matrices $A$ and $B$ :

$$
g \widetilde{T}^{a} g^{-1}=B^{s a}(g) T_{s}+A_{s}^{a}(g) \widetilde{T}^{s} .
$$

Differentiating both sides, expressing $d g$ as $R^{a} T_{a} g$ and using the commutation relations for the Drinfeld double gives

$$
d B^{a b}(g)=R^{s}(g)\left(f_{s t}^{a} B^{t b}-\tilde{f}_{s}^{a t} A_{t}^{b}(g)\right), \quad d A_{b}^{a}(g)=-R^{s}(g) A_{t}^{a}(g) f_{s b}^{t} .
$$

Recalling the definition of the directional derivative

$$
\widetilde{\partial}_{s} f(g)=\left.\frac{d}{d s} f\left(e^{s T^{a}} g\right)\right|_{s=0}=R_{a}^{s}(g) \frac{\partial}{\partial x^{s}} f(g), \quad g=e^{x^{s} T_{s}} \in G
$$

we end up with

$$
\left\{\begin{array}{l}
\widetilde{\partial}_{s} B^{a b}(g)=f_{s t}^{a} B^{t b}(g)-\tilde{f}_{s}^{a t} A_{t}^{b}(g) \\
\widetilde{\partial}_{s} A_{b}^{a}(g)=-A_{t}^{a}(g) f_{s b}^{t}, \quad \widetilde{\partial}_{s} A_{b}^{a}\left(g^{-1}\right)=f_{s t}^{a} A_{b}^{t}\left(g^{-1}\right)
\end{array}\right.
$$

Recalling the definition of the bivector $\Pi^{a b}(g)=-B^{a s}(g) A_{s}^{b}\left(g^{-1}\right)$, we conclude to

$$
\widetilde{\partial}_{s} \Pi^{a b}(g)=\tilde{f}_{s}^{a b}-f_{s t}^{a} \Pi^{b t}(g)+f_{s t}^{b} \Pi^{a t}(g) .
$$

This derivative can also be interpreted as

$$
\widetilde{\partial}_{c} \Pi^{a b}(g)=\tilde{f}_{c}^{a b}(g) \equiv A_{s}^{a}\left(g^{-1}\right) A_{t}^{b}\left(g^{-1}\right) \tilde{f}_{u}^{s t} A_{c}^{u}(g)
$$

To prove this relation, let us start from

$$
\tilde{f}_{u}^{s t}=\left\langle\left[\widetilde{T}^{s}, \widetilde{T}^{t}\right], T_{u}\right\rangle \quad \Rightarrow \quad \tilde{f}_{c}^{a b}(g)=\left\langle\left[A_{s}^{a}\left(g^{-1}\right) \widetilde{T}^{s}, A_{t}^{b}\left(g^{-1}\right) \widetilde{T}^{t}\right], A_{c}^{u}(g) T_{u}\right\rangle .
$$

Using (71) we can write

$$
\tilde{f}_{c}^{a b}(g)=\left\langle\left[g^{-1} \widetilde{T}^{a} g-B^{a u}(g) T_{u}, g^{-1} \widetilde{T}^{b} g-B^{b v}(g) T_{v}\right], g^{-1} \widetilde{T}^{a} g\right\rangle .
$$

Due to the isotropy property only three terms out of four do not vanish. The $A d_{g}$ invariance gives for the first term

$$
\left\langle g^{-1}\left[\widetilde{T}^{a}, \widetilde{T}^{b}\right] g, g^{-1} T_{s} g\right\rangle=\left\langle\left[\widetilde{T}^{a}, \widetilde{T}^{b}\right], T_{s}\right\rangle=\tilde{f}_{c}^{a b} .
$$

The second term is

$$
\begin{aligned}
& =-B^{a u}(g)\left\langle\left[T_{u}, g^{-1} \widetilde{T}^{b} g\right], g^{-1} T_{s} g\right\rangle=-B^{a u}(g)\left\langle\left[g^{-1} T_{u} g, \widetilde{T}^{b}\right], T_{s}\right\rangle= \\
& =-B^{a u}(g) A_{u}^{v}\left(g^{-1}\right)\left\langle\left[T_{v}, \widetilde{T}^{b}\right], T_{s}\right\rangle=\Pi^{a v}(g)\left(-f_{v s}^{b}\right)=f_{s t}^{b} \Pi^{a t}(g),
\end{aligned}
$$

and the third term is just the opposite of the second term with the exchange $(a \leftrightarrow b)$, and this concludes the proof. 


\section{A.2 Identities involving $\Pi$ derivatives}

The transition from $\tilde{f}_{c}^{a b}$ to $\tilde{f}_{c}^{a b}(g)$ amounts to a change of basis in the Lie algebra $\mathcal{G}$. It follows that they do verify the Jacobi identity:

$$
\tilde{f}_{c}^{s t}(g) \tilde{f}_{t}^{a b}(g)+\tilde{f}_{c}^{a t}(g) \tilde{f}_{t}^{b s}(g)+\tilde{f}_{c}^{b t}(g) \tilde{f}_{t}^{s a}(g)=0 .
$$

Contracting the indices $c$ and $s$ we get

$$
\tilde{f}_{s}^{s t}(g) \tilde{f}_{t}^{a b}(g)=0,
$$

which becomes, using relation (76)

$$
\widetilde{\partial}_{s} \Pi^{s t}(g) \widetilde{\partial}_{t} \Pi^{a b}(g)=0 .
$$

Let us notice that

$$
\tilde{f}_{s}^{s a}(g)=\tilde{f}_{s}^{s t} A_{t}^{a}\left(g^{-1}\right) .
$$

Using the last relation in (74) we get

$$
\widetilde{\partial}_{t} \tilde{f}_{s}^{s a}(g)=\tilde{f}_{s}^{s t} f_{t u}^{a} A_{t}^{u}\left(g^{-1}\right)=f_{t u}^{a} \tilde{f}_{s}^{s u}(g),
$$

and from (76) we conclude to the identity

$$
\widetilde{\partial}_{t} \widetilde{\partial}_{s} \Pi^{s a}(g)=f_{t u}^{a} \widetilde{\partial}_{s} \Pi^{s u}(g) .
$$

\section{References}

[1] E. Alvarez, L. Alvarez-Gaumé and Y. Lozano, Nucl.Phys.Proc.Suppl. 41 (1995) 1.

[2] A. Bossard and N. Mohammedi, Nucl. Phys. B 619 (2001) 128.

[3] P. Y. Casteill and G. Valent, Nucl. Phys. B 591 (2000) 491.

[4] B.E. Fridling and A. Jevicki, Phys. Lett. B 134 (1984) 70; E.S. Fradkin and A.A. Tseytlin, Ann. Phys. 162 (1985) 31; X. de la Ossa and F. Quevedo, Nucl. Phys. B 403 (1993) 377.

[5] B. E. Fridling and A. E. M. van de Ven, Nucl. Phys. B, 268 (1986) 719.

[6] C. Klimčík, Commun. Math. Phys., 217 (2001) 203.

[7] C. Klimčík and P. Ševera, Phys. Lett. B 351 (1995) 455.

[8] C. Klimčík and P. Ševera, Phys. Lett. B 372 (1996) 65.

[9] C. Klimčík and G. Valent, Phys. Lett. , B 565 (2003) 237.

[10] K. Kikkawa and M. Yamasaki, Phys. Lett. B 149 (1984) 357.

[11] N. Sakai and I. Senda, Prog. Theor. Phys. 75 (1986) 692.

[12] K. Sfetsos, Phys. Lett. B 432 (1998) 365.

[13] K. Sfetsos, Nucl. Phys. B 517 (1998) 549.

[14] E. Tyurin, Phys. Lett. B 348 (1995) 386. 